\title{
Diseño de un Cuestionario para Valorar los Atributos de Grupos de Interés Universitarios desde un Enfoque de Liderazgo Participativo
}

\author{
Martín A Pantoja(1), María del P. Rodríguez ${ }^{(1)}$ y Andrés Carrión ${ }^{(2)}$ \\ (1) Universidad Nacional de Colombia, Facultad de Ingeniería y Arquitectura, Departamento de Ingeniería \\ Industrial, Campus La Nubia. Manizales-Colombia (e-mail: mapantojao@unal.edu.co, \\ mdrodriguezco@unal.edu.co) \\ (2) Universidad Politécnica de Valencia, Centro de Gestión de la Calidad y del Cambio. Valencia-España \\ (e-mail: acarrion@eio.upv.es)
}

Recibido Nov. 6, 2014; Aceptado Dic. 12, 2014; Versión final recibida Feb. 26, 2015

\begin{abstract}
Resumen
En este artículo se validan los constructos de un cuestionario propuesto para valorar los atributos (poder, legitimidad y urgencia) de grupos de interés universitarios. Desde una perspectiva subjetiva y un enfoque de relaciones, se recogió la percepción de una muestra de líderes de universidades públicas de Manizales (Colombia). Se conformaron dos grupos de constructos; el primero recoge los atributos de los grupos de interés universitarios y el segundo recoge la relevancia de éstos. Los resultados de los alfa de Cronbach indican que es factible medir de manera eficaz los constructos propuestos. Se concluye que, desde el nivel de análisis individual, el cuestionario propuesto cuenta con la coherencia interna y la fiabilidad de medida necesaria para valorar los atributos de los grupos de interés universitarios. En este análisis se considera que el contexto organizacional determina sus grupos de interés y que la percepción de los líderes determina la relevancia de los atributos.
\end{abstract}

\section{Design of a Questionnaire to Assess University Stakeholders Attributes from a Participative Leadership Approach}

\begin{abstract}
In this paper the constructs of a proposed questionnaire that assess the university stakeholders attributes (power, legitimacy and urgency) are validated. From a subjective perspective and a relational approach, the perceptions of a sample of public universities leaders from Manizales (Colombia) were collected. Two groups of constructs were formed, the first includes the university stakeholders attributes mentioned above and the second collects their relevance. The Cronbach alpha values indicate that is feasible to measure effectively the proposed constructs. It is concluded that, from an individual level of analysis, the proposed questionnaire has the internal consistency and the measure reliability for assessing the university stakeholders attributes. In this analysis, it is considered that the organizational context determines its stakeholders and that leaders' perceptions determine the relevance of the attributes.
\end{abstract}

Keywords: university stakeholders, participative leadership, attributes assessment 


\section{INTRODUCCIÓN}

Referirse al liderazgo implica necesariamente hablar de la relevancia e influencia de los grupos de interés de la organización. Ignorar esta relación significaría negar la naturaleza sistémica de las organizaciones e incluso la del liderazgo mismo pues la interdependencia entre los grupos de interés internos y externos de la organización es una realidad, como lo afirman Vroom y Jago (1995) y Porter y McLaughlin (2006) para quienes el liderazgo es contextual. Las organizaciones están enmarcadas en un contexto conformado por actores internos y actores externos quienes tienen la capacidad para influir en los procesos que se lleven a cabo en la organización. El liderazgo en general y el líder que ocupa un cargo directivo en particular, no son ajenos a esta condición.

Desde una perspectiva ampliada existe una relación de intercambio entre las manifestaciones de los procesos internos y la influencia de los diferentes actores (Mintzberg, 1983) o grupos de interés (Freeman, 2001), cuyos atributos básicos: poder, legitimidad y urgencia (Mitchell et al., 1997) son sus instrumentos de influencia. Uno de estos procesos internos es el ejercicio del liderazgo participativo en el cual un líder, como ocupante de un cargo directivo, otorga grados crecientes de autonomía y participación a sus colaboradores para que influyan en la toma de decisiones (Yukl, 2010). Sin embargo, las relaciones entre la relevancia de los atributos de los grupos de interés y la forma como se manifiesta el liderazgo en general y la participación en particular, han recibido escasa atención en la literatura científica (Mitchell et al., 1997; Schneider, 2002; Myllykangas et al., 2010). El liderazgo no sucede en el vacío pues la organización como tal existe enmarcada dentro de un contexto que la contiene (Katz y Kahn, 1977; Osborn et al., 2002). De acuerdo con Porter y McLaughlin (2006) el contexto, que no ha recibido la atención que su importancia merece para la comprensión del fenómeno del liderazgo, lo conforman los grupos de interés de la organización (Freeman, 2001) quienes en sus interacciones, forman relaciones sociales de intercambio entre los involucrados (Homans, 1961; Blau, 1964).

Particularmente, dependiendo de la valoración que haga el líder de los atributos que posee un grupo de interés le asigna una relevancia y con base en ésta, ese grupo puede o no convertirse en sujeto de su atención. Estos atributos son percibidos por el líder, procesados y al final, de acuerdo con Katz y Kahn (1977), manifestados en comportamientos. De esta forma los grupos de interés se convierten en actores no sólo con poder, sino también con la legitimidad y urgencia necesarias como para condicionar la actuación del líder en la organización, pues al transmitir sus expectativas al líder terminan influyendo en las conductas que manifiesta en su calidad de persona focal (Katz y Kahn, 1977). En consecuencia, el liderazgo en la organización está construido en los grupos de interés y para los grupos de interés, por lo que se hace necesario, como aproximación inicial, diseñar un cuestionario y validar los constructos que permitan valorar los atributos de los grupos de interés desde la percepción del líder en un nivel de análisis individual.

La atención al contexto, pero especialmente, a los actores (grupos de interés) que rodean el ejercicio del liderazgo, ha recibido poca atención y los desarrollos que lo consideran como relevante e influyente refieren propuestas de carácter teórico que no han sido implementadas en la práctica. Se necesitan modelos congruentes y sistemáticos que sienten las bases para llevar a cabo procesos de investigación aplicada, que permitan identificar y describir de mejor forma el fenómeno de las relaciones de intercambio que se presentan entre un líder, como ocupante de un cargo directivo, y sus grupos de interés. Este es el marco básico desde el cual se enfoca el desarrollo del cuestionario a validar, en cumplimiento de una de las etapas de la investigación a la cual pertenece y que, como objetivo general, busca explicar las relaciones de influencia entre los atributos de los grupos de interés y las expresiones del liderazgo participativo en la organización. Sus objetivos específicos buscan primero, construir la discusión teórica que identifique y describa las relaciones existentes entre el proceso de liderazgo participativo y los atributos que hacen relevantes a los grupos de interés en una organización; segundo, proponer un modelo que dé cuenta de las relaciones encontradas y tercero, ilustrar la aplicación del modelo propuesto para un contexto organizacional colombiano particular.

El cuestionario propuesto, contribuye al cumplimiento del segundo objetivo específico y permite identificar el marco general dentro del cual se inserta el modelo a desarrollar, específicamente en la intersección de la relación entre los locus del contexto y del líder con el mecanismo de los comportamientos. Para construirlo, se tuvo en cuenta la propuesta de los autores del desarrollo del Modelo de Relaciones de Intercambio LíderStakeholder o SLX, el cual se sustenta en el planteamiento de los locus y los mecanismos del liderazgo formulada por Hernandez et al. (2011). Adicionalmente, y desde la perspectiva de la relación que proponen Graen y Uhl-Bien (1995), se llevó a cabo una adaptación que permite conducir el análisis de esta relación de intercambio entre el líder y sus grupos de interés desde el nivel de análisis individual, y particularmente, desde la percepción del líder. 
Diferentes autores han estudiado el liderazgo en organizaciones educativas y algunos coinciden en afirmar que las estructuras influyen y condicionan las acciones de la organización y además, que el liderazgo puede surgir de cualquier lugar, particularmente de actores internos (Torres et al., 2011) o que la situación crea a los líderes (Delgado et al., 2011) quienes normalmente ocupan cargos directivos (Reche et al., 2008; Torres et al., 2011; Delgado, 2012). Aplicados a este mismo contexto otros autores han desarrollado instrumentos de medida del liderazgo, tales como Delgado et al. (2011), cuyo cuestionario mide las percepciones respecto de cómo se desarrollan las prácticas de liderazgo y el de Gastélum et al. (2012), diseñado para identificar algunas conductas de liderazgo. En ambos casos, los cuestionarios hicieron parte de diseños no experimentales haciendo uso del método de encuesta para registrar las percepciones del líder.

Dada la importancia de sustentar el cuestionario desarrollado, la revisión teórica correspondiente a este aspecto será tratada en detalle en el apartado siguiente titulado Revisión y Antecedentes. En particular en la literatura sobre liderazgo, son escasos los abordajes de relaciones con los grupos de interés, y en términos de método la referencia que se hace es escasa. Solo se aproxima la propuesta del modelo de los Grupos de Interés para el Liderazgo Organizacional sugerido por Schneider (2002). El tipo de investigación dentro del cual se enmarca el estudio al cual pertenece el cuestionario diseñado, es de carácter explicativo (Toro y Parra, 2010) y aplicado, en concordancia con su objetivo general, aunque también cuenta con un trasfondo que incluye aspectos relacionados con la exploración y la descripción. La originalidad de su contribución se sugiere que aportará evidencia nueva dentro de un problema viejo (Phillips y Pugh, 2008) y su novedad radica en la identificación de la influencia que los atributos de los grupos de interés ejercen sobre las expresiones del liderazgo participativo en la organización. Obedeciendo a un diseño de corte noexperimental de tipo transeccional o transversal (Toro y Parra, 2010), el método que guía el proceso de investigación es el hipotético-deductivo. Para llevar a cabo la prueba piloto del cuestionario se seleccionó a los ex directivos de las universidades públicas de la ciudad de Manizales (Departamento de Caldas) en Colombia. Los resultados indican que, en su versión de autoevaluación y acorde con el nivel de análisis individual seleccionado, el cuestionario diseñado resulta válido y confiable para valorar los atributos de los grupos de interés universitarios en las organizaciones estudiadas.

\section{REVISIÓN Y ANTECEDENTES}

En términos generales, el liderazgo se ha entendido como la capacidad para influir en el comportamiento de otros con el propósito de alcanzar un resultado (Bass, 2008; Yukl, 2010), a lo que Pareja Fernández de la Reguera et al. (2012) agregan, que esta influencia se basa en la aceptación de los principios que se proponen como premisas para la acción. Un directivo de una organización está en la obligación de encaminar la intención y las actividades de sus dirigidos hacia un determinado fin, y es mediante el uso de su capacidad para influir, esto es, para liderar, como logra encaminar la intención y las actuaciones de sus dirigidos. Así, la influencia que puede ejercer un directivo, se encuentra apalancada por la posición de rango superior que ocupa en la estructura formal de la organización.

En la tradición científica se han postulado diferentes tipos de liderazgo. Así por ejemplo, el liderazgo carismático y transformacional apela a los valores morales y enfatiza en los procesos emocionales, además incluye comunicar la visión para inspirar a los seguidores, en tanto que las teorías éticas del liderazgo, que incluyen el liderazgo de servicio, el espiritual y el auténtico, consideran los valores, rasgos y comportamientos del líder y se interesan en el riesgo que implica el uso inadecuado del poder (Bass, 2008; Yukl, 2010). Por su parte el denominado liderazgo pedagógico busca mejorar la calidad de las relaciones profesor, estudiante, equipo directivo, en donde el líder desempeña un papel mediador entre estos actores (Delgado et al., 2011). Específicamente, para la investigación a la cual pertenece el cuestionario a validar, el liderazgo participativo involucra el esfuerzo del líder por impulsar y abrir la puerta a la participación de otros en la toma de decisiones importantes (Yukl, 2010), concepto que, aunque guarda semejanzas con otros como el de liderazgo distribuido que adoptan Pareja Fernández de la Reguera et al. (2012), se diferencia de éste en el sentido que no busca expresamente vincular las denominadas fuentes informales de liderazgo en la estructura formal de la organización. Esto debido a que liderazgo participativo considera que la influencia de otros en la toma de decisiones del líder, es vinculada por éste cuando manifiesta las cuatros expresiones que lo conforman, las cuales reciben el influjo de los atributos de los grupos de interés, tal como lo plantea el objetivo general de la investigación a la que pertenece el cuestionario aquí propuesto.

Como marco interpretativo que sostiene los planteamientos aquí contenidos, el artículo que se presenta surge de adoptar la perspectiva subjetiva de investigación la cual toma en cuenta al sujeto y al contexto dentro del cual se encuentra inmerso. Así pues, el mundo será conocido con base en las interpretaciones que los sujetos hacen de él y, en particular, aquellas de los líderes en la organización (May, 2011). Además, y con base en Searle (1997), la organización, los grupos de interés y sus atributos (poder, legitimidad y urgencia), el liderazgo, el liderazgo participativo y sus expresiones (autocracia, consulta, decisión conjunta, 
delegación) son ontológicamente subjetivos debido a que su existencia y significado en el mundo dependen indisolublemente de la percepción que los sujetos tienen de esos fenómenos.

Reconociéndolos como fenómenos subjetivos que se interrelacionan y se correlacionan en el contexto de la organización, en este artículo se consideran como hechos sociales que son epistemológicamente objetivos, en razón a que su interpretación y significado no dependen de la percepción de un sujeto singular aislado, sino de la interpretación y significado aceptados y compartidos que les otorgan los sujetos en su conjunto; significado que depende de una intencionalidad colectiva (Searle, 2005) basada en reglas constitutivas establecidas (Searle, 1997). Siendo los atributos de los grupos de interés (poder, legitimidad, urgencia) una realidad del mundo 3 (Popper, 1994), entonces a semejanza de la forma como se configura la realidad, de igual forma se configura la influencia de esos atributos sobre los habitantes de la organización en donde la realidad que se busca describir es aquella que percibe el líder en la organización.

En relación con las metodologías para el estudio del liderazgo, estas se tornan variadas, tal como el campo mismo. No existe una metodología que prevalezca sobre otras para llevar a cabo investigaciones en el campo del liderazgo. Tal como no existe una única e integral definición de liderazgo, tampoco existe una única e integral metodología que permita satisfacer todas las necesidades que los investigadores tienen respecto del liderazgo y sus implicaciones. El campo, según Gardner et al. (2010) es, además de diverso y robusto, poseedor de múltiples facetas, focos y niveles, a lo que añaden Stentz et al. (2012) su naturaleza compleja y socialmente construida.

En cuanto al diseño, Antonakis et al. (2004) plantean que este se encuentra ligado al tipo de investigación que se requiera desarrollar. Particularmente, en el campo del liderazgo han predominado los diseños cuantitativos en una proporción de cuatro a uno con respecto a los cualitativos (Gardner et al. 2010; Stentz et al., 2012), aunque una combinación incrementa la validez de los resultados (Bass, 2008; Stentz et al., 2012). Según Antonakis et al. (2004) los diseños cuantitativos se usan cuando el fenómeno de interés necesita ser medido y su fortaleza radica en su capacidad para evaluar la teoría. Los de tipo cualitativo son más utilizados cuando de construir un marco complejo y holístico se trata. Se le atribuye a este último la cualidad de permitir entender de mejor forma un fenómeno complejo y de naturaleza contextual y que para el caso del liderazgo, restringe la variabilidad que potencialmente puede ser medida, evitando conclusiones erradas que pueden surgir al pasar por alto el contexto dentro del cual se enmarca el fenómeno.

Entre los factores contextuales a los que se refieren Antonakis et al., (2004) destaca como uno de los principales al nivel de análisis, siendo este un aspecto que dentro de la tradición literaria en liderazgo ha venido ganando una amplia importancia (Yammarino et al., 2005). Debido a que el interés en materia de liderazgo se centra en los seres humanos que se congregan en las organizaciones (Yammarino et al., 2005), estos niveles de análisis están directamente relacionados con el locus de los fenómenos que se presentan cuando los seres humanos se congregan y trabajan juntos en las organizaciones modernas. Organizacionalmente estos niveles de análisis se manifiestan de cuatro formas diferentes: individuo, díada, grupo y organización y cuyas unidades de análisis las componen respectivamente: personas y seres humanos independientes para el primero; grupos de dos personas y relaciones interpersonales para el segundo; grupos y equipos de trabajo para el tercero y colectivos más amplios que los grupos y grupos de grupos para el cuarto (Schriesheim et al, 2001; Yammarino et al., 2005; Dionne et al., 2012).

En las investigaciones en liderazgo se hace necesario que los momentos de teorización, medición y análisis permanezcan alineados con el nivel de análisis escogido a fin de guardar la congruencia que posibilite la generación de resultados válidos y confiables de modo que las asociaciones teóricas que se desarrollen resulten pertinentes y valiosas (Yammarino et al., 2005). En este orden de ideas, las implicaciones del uso de los niveles de análisis en la investigación en liderazgo adquiere la categoría de variable moderadora o mediadora para explicar los fenómenos en diferentes enfoques de liderazgo (Yammarino et al., 2005).

En particular para la investigación de tipo mixto que nos ocupa, se tienen en cuenta los dominios del liderazgo (Graen y Uhl-Bien, 1995) que condujeron a la formulación de una relación emergente entre el líder y sus grupos de interés, la cual necesita ser estudiada dentro del marco de los niveles de análisis para el estudio del liderazgo (Schriesheim et al., 2001; Yammarino et al., 2005; Dionne et al., 2012). Dentro del estudio al cual pertenece el cuestionario a validar, se analiza la relación desde el nivel y percepción del líder, esto es, desde el nivel de análisis individual.

Así pues, dentro del nivel de análisis individual seleccionado, la unidad de análisis será la persona del líder en un cargo directivo, en lo que tiene que ver con sus percepciones como persona focal respecto de la influencia que proviene de sus grupos de interés como emisores del papel (Katz y Kahn, 1977) y que viene mediada por la valoración que hace de la relevancia de sus atributos (poder, legitimidad y urgencia). Acto seguido, se despliega la conducta del papel del líder como persona focal, cuyo elemento de interés es su 
comportamiento como directivo, manifestado en las diferentes expresiones del liderazgo participativo (autocracia, consulta, decisión conjunta, delegación) tal como se ilustra en la Figura 1, en la cual se aprecian las características generales del modelo que los autores desarrollan, y que en su primer momento, se refiere a la percepción del líder en un cargo directivo respecto de la influencia de los atributos de los grupos de interés, cuyo instrumento de medición es el objeto de la validación a la que se refiere el presente artículo.

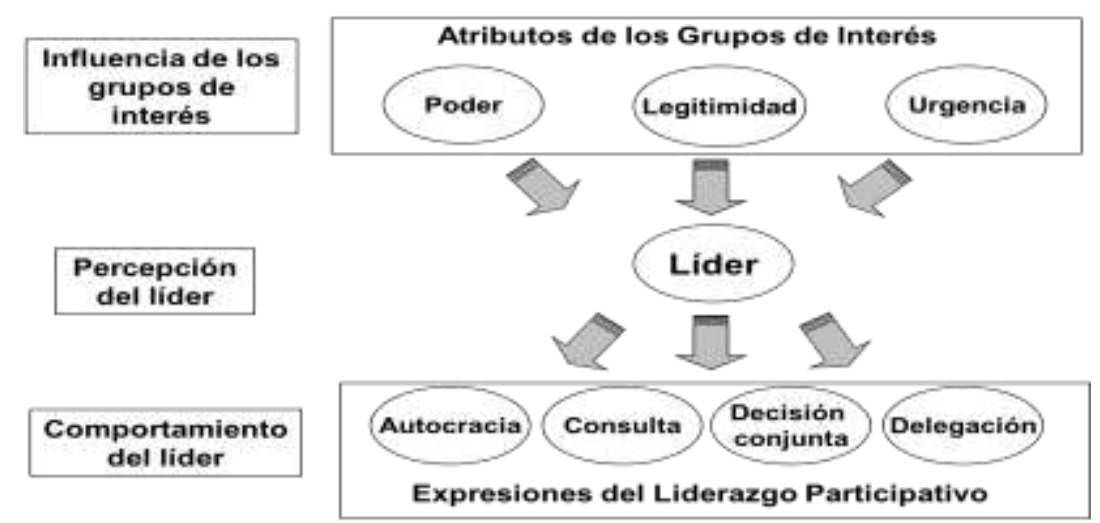

Fig. 1: Influencia de los atributos de los grupos de interés sobre el líder

\section{MATERIALES Y MÉTODOS}

Para contribuir al cumplimiento del tercer objetivo específico de la investigación a la que pertenece el cuestionario a validar, se seleccionó el contexto de las universidades públicas de la ciudad de Manizales (Colombia), debido a que el tamaño y cobertura de su estructura y oferta académica genera un significativo impacto en los grupos de interés que conforman su contexto, si se le compara con el conjunto de universidades privadas de esta misma ciudad. Adicionalmente, existen antecedentes investigativos sobre los grupos de interés de estas universidades públicas, los cuales han sido usados como insumo (Vieira, 2013)

La población actual de líderes ocupantes de cargos directivos de las tres universidades que son de interés para el estudio, asciende a 121. Mediante la técnica de muestreo por conveniencia, y tal como se ha hecho para otras investigaciones de liderazgo en las universidades (Reche et al., 2009), se seleccionó a quienes harían parte de la fase piloto, considerando todos los niveles de la estructura orgánica interna y que fueran representantes de las diferentes instancias académicas y administrativas. En cumplimiento de los objetivos de diseñar un cuestionario que valore los atributos de los grupos de interés y su correspondiente validación, se llevaron a cabo seis etapas (ver Tabla 1). Algunos de los elementos teóricos a los que se refieren las etapas 1 y 2, han sido incluidos en la discusión teórica previa en este artículo, en tanto la Figura 1 da cuenta de las relaciones que el modelo de la etapa 3 plantea. Las restantes dos etapas se discuten a continuación.

Tabla 1: Etapas de la metodología procesal

\begin{tabular}{|c|l|}
\hline Etapa & \multicolumn{1}{|c|}{ Atributos de los grupos de interés } \\
\hline Etapa 1 & $\begin{array}{l}\text { Sustentar teóricamente el poder, la legitimidad y la urgencia, como atributos que hacen relevantes } \\
\text { e influyentes a los grupos de interés. }\end{array}$ \\
\hline Etapa 2 & $\begin{array}{l}\text { Identificar y describir teóricamente las relaciones entre el proceso de liderazgo participativo, } \\
\text { incluidas sus expresiones, y los atributos que hacen relevantes a los grupos de interés. }\end{array}$ \\
\hline Etapa 3 & $\begin{array}{l}\text { Diseñar un modelo que explique las relaciones entre el proceso de liderazgo participativo, incluidas } \\
\text { sus expresiones, y los atributos que hacen relevantes a los grupos de interés. }\end{array}$ \\
\hline Etapa 4 & $\begin{array}{l}\text { Diseñar el cuestionario que permita valorar los atributos de los grupos de interés en una } \\
\text { organización. }\end{array}$ \\
\hline Etapa 5 & Validar los constructos del cuestionario \\
\hline
\end{tabular}

\section{Diseño del cuestionario}

En el diseño del cuestionario para valorar los atributos de los grupos de interés, se tomó como punto de partida la teoría del intercambio social, la cual se ocupa tanto de las relaciones como de las obligaciones que comparten ambas partes en una relación (Homans, 1961). De acuerdo con Morales Rodríguez (1978) esta relación tiene su origen en el propio interés, y además, como fenómeno social, se hace necesario 
explicarlo tomando como unidad de análisis al individuo. Adicionalmente, y de acuerdo con Homans (1961), la autoridad depende fundamentalmente de la cantidad de influencia que pueda ser ejercida por parte de una persona sobre otras y también sugiere que en la medida en que se otorgan más recompensas dentro de un amplio rango de actividades específicas, en la misma medida se amplían, tanto la autoridad como el rango dentro del cual ésta puede ejercerse.

De acuerdo con lo anterior, es válido considerar que los grupos de interés con más autoridad serán aquellos que, o bien tienen una estrecha relación con el líder, o bien tienen unos atributos que les dan más relevancia y, por lo tanto, más influencia sobre el líder en la organización. Aquí conviene destacar que, dentro de la teoría del intercambio social, Blau (1964) planteó que las conductas que buscan el intercambio necesariamente son intencionales, a diferencia de los postulados inicialmente propuestos por Homans (1961) para quien todas las relaciones sociales son de intercambio sin diferenciar las intencionales de aquellas que no lo son. En este sentido, es admisible considerar que cuando los grupos de interés tienen una relación de intercambio con el líder, ésta tiene una intención, además de que existen, de acuerdo con Morales Rodríguez (1978), dos características que desestabilizan la relación de intercambio: el estatus y el poder, los cuales se pueden asociar a los atributos de legitimidad y poder, respectivamente.

En este orden de ideas, se entiende el concepto de intensidad planteado por Homans (1961) como el grado de fuerza con que se manifiesta la cualidad de los grupos de interés de ser influyentes sobre el líder, específicamente, mide la magnitud de la capacidad de esa influencia. Por su parte, el concepto de relevancia planteado por Mitchell et al. (1997) se puede entender como la cualidad de un grupo de interés de ser sobresaliente, importante o significativo para el líder. Se tiene entonces que la intensidad de los intentos de influencia de un grupo de interés sobre un líder, es equivalente a la relevancia combinada de los atributos (poder, legitimidad y urgencia) del mismo grupo de interés.

Existe entonces equivalencia semántica y conceptual entre esa intensidad con la relevancia que un grupo de interés puede tener sobre el líder. Por lo tanto cuando se habla de la intensidad de los intentos de influencia de los grupos de interés de la organización sobre el líder, este concepto se puede entender como la relevancia que un grupo de interés puede tener sobre un líder. Así pues, en el diseño del cuestionario para valorar los atributos de los grupos de interés, se ha tomado como base el planteamiento de los atributos (poder, legitimidad y urgencia) propuesto por Mitchell et al. (1997) y que ha sido validado por estudios recientes (Magness, 2008; Myllykangas et al., 2010); y con el objetivo de hacer congruente la medición con el modelo planteado en la fase 3, se incorpora la variable frecuencia de los intentos de influencia, que originalmente también fue sugerida por Homans (1961) al referirse a la intensidad y frecuencia de los intentos de influencia.

En el proceso de estructuración del cuestionario definitivo, y de forma semejante a como lo hicieran AyugaTéllez et al. (2012) y Reche et al. (2008), fueron consultados dos expertos pertenecientes al Grupo de Investigación Ethos de la Universidad Nacional de Colombia y un tercer miembro de este grupo experto en grupos de interés y temas afines, quien en un uno de sus estudios previos propuso una taxonomía para la clasificación de los grupos de interés universitarios (Vieira, 2013) cuyo planteamiento también fue acogido pues consolida las propuestas de clasificación de grupos de interés universitarios de diferentes autores (Duque Oliva, 2009; Vallaeys et al., 2009; Rodríguez Fernández, 2010).

\section{Estructura del cuestionario}

El cuestionario fue diseñado para ser usado con entrevistador. Las preguntas son cerradas, con escala verbal de valoración de cuatro niveles, semejante a otras ya usadas en investigaciones sobre liderazgo (Reche et al., 2008; Delgado et al., 2011). Esta escala avanza desde nula (cero puntos) hasta alta (tres puntos) para reflejar la influencia de cada atributo o la frecuencia de los intentos de influencia y se escogió para evitar el riesgo de tendencia central que suele suceder con escalas de nivel impar en las cuales, la indecisión o la premura del entrevistado pueden conducirlo al centro de la escala. Una ventaja de esta escala es permitir una decisión en menos tiempo, con mayor facilidad y comprendiendo fácilmente lo que se busca, que indique su selección entre alto, medio, bajo o nulo para valorar la intensidad de los atributos.

La población a la cual va dirigido el cuestionario, está conformada por los líderes directivos de las tres universidades públicas de la ciudad de Manizales (Colombia). En las instrucciones del cuestionario (ver Figura 2) se le pide a cada entrevistado que, tomando en cuenta las definiciones para los atributos que hacen relevantes a los grupos de interés, señalen en la escala correspondiente la influencia que cada uno de esos atributos pudo tener sobre su comportamiento como directivo de la universidad. Esas definiciones fueron tomadas según los planteamientos de Mitchell et al. (1997): 
- el poder es la capacidad de un grupo de interés para hacer que alguien haga algo que de otra forma no estaría dispuesto a hacer,

- la legitimidad es la percepción generalizada o presunción de que las acciones de un grupo de interés son deseables, correctas o apropiadas, teniendo en cuenta las normas, valores, creencias y definiciones propias de la Universidad,

- la urgencia se define como el grado en el cual un grupo de interés reclama ser atendido de forma inmediata, teniendo en cuenta la sensibilidad al tiempo y la criticidad de su exigencia.

Finalmente y teniendo en cuenta que los grupos de interés pueden contactar a los líderes directivos de diferentes formas (una visita personal, una llamada telefónica, una carta o correo electrónico, o un mensaje por intermedio de otras personas o grupos que los representen, entre otros), se les pidió que valoraran la frecuencia de los intentos de influencia de sus grupos de interés sobre él.

\begin{tabular}{|c|c|c|c|c|c|c|c|c|c|c|}
\hline \multirow{3}{*}{ \# } & \multirow{3}{*}{$\begin{array}{l}\text { Grupo de interés } \\
\text { universitario }\end{array}$} & \multirow{3}{*}{ Atributo } & \multicolumn{4}{|c|}{ Escala de Influencia } & \multicolumn{4}{|c|}{ Frecuencia de los intentos de influencia } \\
\hline & & & Alta & Media & Baja & Nula & Alta & Media & Baja & Nula \\
\hline & & & 3 & 2 & 1 & 0 & 3 & 2 & 1 & 0 \\
\hline \multirow{3}{*}{1} & \multirow{3}{*}{$\begin{array}{l}\text { Directivas de la } \\
\text { Universidad }\end{array}$} & Poder & $\square$ & $\square$ & $\square$ & $\square$ & & & & \\
\hline & & Legitimidad & $\square$ & $\square$ & $\square$ & $\square$ & & & & \\
\hline & & Urgencia & $\square$ & $\square$ & $\square$ & $\square$ & & & & \\
\hline \multirow{3}{*}{2} & \multirow{3}{*}{ Profesores } & Poder & $\square$ & $\square$ & $\square$ & $\square$ & & & & \\
\hline & & Legitimidad & $\square$ & $\square$ & $\square$ & $\square$ & & & & \\
\hline & & Urgencia & $\square$ & $\square$ & $\square$ & $\square$ & & & & \\
\hline \multirow{3}{*}{3} & \multirow{3}{*}{$\begin{array}{l}\text { Grupos de } \\
\text { Investigación }\end{array}$} & Poder & $\square$ & $\square$ & $\square$ & $\square$ & & & & \\
\hline & & Legitimidad & $\square$ & $\square$ & $\square$ & $\square$ & & & & \\
\hline & & Urgencia & $\square$ & $\square$ & $\square$ & $\square$ & & & & \\
\hline \multirow{3}{*}{4} & \multirow{3}{*}{ Empresas } & Poder & $\square$ & $\square$ & $\square$ & $\square$ & & & & \\
\hline & & Legitimidad & $\square$ & $\square$ & $\square$ & $\square$ & & & & \\
\hline & & Urgencia & $\square$ & $\square$ & $\square$ & $\square$ & & & & \\
\hline \multirow{3}{*}{5} & \multirow{3}{*}{ Estudiantes } & Poder & $\square$ & $\square$ & $\bar{\square}$ & $\bar{\square}$ & & & & \\
\hline & & Legitimidad & $\square$ & $\square$ & $\square$ & $\square$ & & & & \\
\hline & & Urgencia & $\square$ & $\square$ & $\square$ & $\square$ & & & & \\
\hline \multirow{3}{*}{6} & \multirow{3}{*}{$\begin{array}{l}\text { Sociedad en } \\
\text { general }\end{array}$} & Poder & $\square$ & $\square$ & $\square$ & $\square$ & & & & \\
\hline & & Legitimidad & $\square$ & $\square$ & $\square$ & $\square$ & & & & \\
\hline & & Urgencia & $\square$ & $\square$ & $\square$ & $\square$ & & & & \\
\hline \multirow{3}{*}{7} & \multirow{3}{*}{ Financiadores } & Poder & $\square$ & $\square$ & $\square$ & $\square$ & & & & \\
\hline & & Legitimidad & $\square$ & $\square$ & $\square$ & $\square$ & & & & \\
\hline & & Urgencia & $\square$ & $\square$ & $\square$ & $\square$ & & & & \\
\hline \multirow{3}{*}{8} & \multirow{3}{*}{ Gobierno } & Poder & $\square$ & $\square$ & $\square$ & $\square$ & & & & \\
\hline & & Legitimidad & $\bar{\square}$ & $\square$ & $\bar{\square}$ & $\bar{\square}$ & & & & \\
\hline & & Urgencia & $\square$ & $\square$ & $\square$ & $\square$ & & & & \\
\hline \multirow{3}{*}{9} & \multirow{3}{*}{ Graduados } & Poder & $\square$ & $\square$ & $\square$ & $\square$ & & & & \\
\hline & & Legitimidad & $\square$ & $\square$ & $\square$ & $\square$ & & & & \\
\hline & & Urgencia & $\square$ & $\square$ & $\square$ & $\square$ & & & & \\
\hline \multirow{3}{*}{10} & \multirow{3}{*}{ Medio Ambiente } & Poder & $\square$ & $\square$ & $\square$ & $\square$ & & & & \\
\hline & & Legitimidad & $\square$ & $\square$ & $\square$ & $\square$ & & & & \\
\hline & & Urgencia & $\square$ & $\square$ & $\square$ & $\square$ & & & & \\
\hline \multirow{3}{*}{11} & & Poder & $\square$ & $\square$ & $\square$ & $\square$ & & & & \\
\hline & & Legitimidad & $\square$ & $\square$ & $\square$ & $\square$ & & & & . \\
\hline & & Urgencia & $\bar{\square}$ & $\square$ & $\bar{\square}$ & $\bar{\square}$ & & & & \\
\hline
\end{tabular}

Fig. 2: Esquema general del cuestionario

Se pregunta directamente en el cuestionario por diez diferentes grupos de interés (internos: directivas de la universidad, profesores, grupos de investigación y estudiantes; y externos: empresas, sociedad en general, financiadores, gobierno, graduados y medio ambiente). En la casilla once, se da la opción de seleccionar uno de los siguientes grupos de interés: medios de comunicación, organizaciones no gubernamentales, políticos, administrativos de la universidad, otro (¿cuál?). Conviene aclarar, tal como se hizo a los líderes directivos entrevistados, la diferencia existente entre los grupos de interés directivas de la universidad y gobierno y la precisión sobre el grupo de interés sociedad en general. Las directivas representan el grupo de rango jerárquico superior al interior de cada universidad (rector, vicerrector, decano, director de unidad académica y similares), en tanto el gobierno hace referencia al grupo que ejerce autoridad y control sobre la universidad en representación de la Nación, específicamente la rama ejecutiva del poder público y los órganos de control del Estado Colombiano. Por su parte, a la sociedad en general pertenecen todos los demás actores que afectan o son afectados por las acciones de la universidad y cuya identidad e importancia pueden resultar claras y diferenciables para el líder directivo, pero debido a que son grupos minoritarios o de escaso contacto e interacción directa con la universidad en este momento, no alcanzan a tener la relevancia que efectivamente se reconoce a los otros grupos de interés. 


\section{Prueba piloto}

Con el fin de no desgastar la población objetivo del estudio final, para esta prueba piloto se decidió seleccionar a un conjunto de líderes ex directivos de las tres universidades que hubieran desempeñado recientemente cargos como: Rector, Vicerrector, Decano, Director de Departamento, Director de Área Curricular, Director de Investigación, entre otros. Para el caso de dos universidades fue posible localizar dentro de las mismas a treinta y cuatro ex directivos, los cuales todos se desempeñan en la carrera docente. En la restante fueron ubicados tres ex Directores Regionales y un Director Administrativo y Financiero, todos ellos retirados de esa universidad.

Resulta importante señalar algunas limitantes que se presentaron en esta etapa. En primer lugar la muestra alcanzó un tamaño de treinta y ocho, y aunque hubiera sido deseable ampliarla, el tiempo, la disposición de los ex directivos y su dispersión no hicieron esto posible. En todo caso, dado el carácter de prueba piloto se considera suficiente ese número. En segundo lugar, existen escasos estudios sobre los cuales tomar un referente. Finalmente, podría pensarse que la condición de ex directivos de los participantes pueda estar ofreciendo una percepción que no resulta vigente para el momento en que fueron abordados. Sin embargo, la mayoría, como ya se señaló, son profesores de carrera en dos de las tres universidades y su experiencia y trayectoria les permite captar una percepción ajustada al momento en que fueron entrevistados (adicionalmente, varios de ellos han vuelto a ocupar posiciones directivas después de las entrevistas).

Bajo la técnica de entrevista estructurada estandarizada con preguntas cerradas de valoración, se abordó individualmente a cada ex directivo. Se le exponían los objetivos del estudio y la estructura del cuestionario. Acto seguido, se le pedía que leyera detenidamente las instrucciones y definiciones. Antes de diligenciar las respuestas se resolvían las inquietudes relacionadas con la comprensión de los conceptos teóricos para los atributos (poder, legitimidad y urgencia) y la definición para la variable frecuencia de los intentos de influencia. En el transcurso del diligenciamiento del cuestionario se suministró el acompañamiento y apoyo que cada ex directivo necesitaba para responder con certeza. Una vez diligenciado el cuestionario por parte del ex directivo, el entrevistador verificó que hubiese sido diligenciado completo y correctamente.

Dentro de este proceso de entrevistas a los ex directivos, fue necesario profundizar y aclarar el concepto presentado en el cuestionario para el atributo del poder. Se utilizó el siguiente enfoque: Aunque el poder es complejo de definir, no resulta tan difícil de reconocer pues sus fuentes pueden ser de naturaleza coercitiva, utilitaria o normativa. El poder que un actor tiene dentro de una relación social se asocia a su capacidad para hacer valer su voluntad, aun cuando otro y otros actores en la relación puedan resistirse a ella. Así pues, y en términos particulares, puede entenderse el poder como la capacidad de un grupo de interés para hacer que alguien haga algo que de otra forma no estaría dispuesto a hacer.

\section{RESULTADOS Y DISCUSIÓN}

Sobre la base de datos se han establecido dos tipos de constructo ortogonales entre sí: el primero define cada atributo como un constructo; el segundo define cada grupo de interés como un constructo. Así se tienen un total de 14 constructos: los tres atributos (cada uno en todos los grupos de interés) y los once grupos de interés (incluyendo los tres atributos de grupo, los cuales constituyen su relevancia).

Con el fin de medir la consistencia interna y estimar la fiabilidad del instrumento de medida, se calcularon los alfas de Cronbach para ambos grupos de constructos. Los valores mínimos recomendados para el alfa de Cronbach pueden ser superiores a 0.5 , considerados deseables, y superiores a 0.7 , considerados óptimos (Helmstadter, 1964; Nunnally y Bernstein, 1999). La Tabla 2 muestra los resultados para el constructo atributos (poder, legitimidad y urgencia). Los resultados indican que la medida es aceptable para cada uno de los tres atributos. Aunque se destaca un poco más el atributo de la urgencia que el poder y la legitimidad, el resultado en su conjunto es indicativo de que el cuestionario permite valorar cada uno de ellos en los diferentes grupos de interés considerados.

Tabla 2: Alfas de Cronbach para el constructo atributos

\begin{tabular}{|c|c|}
\hline Poder & 0,758 \\
\hline Legitimidad & 0,693 \\
\hline Urgencia & 0,799 \\
\hline
\end{tabular}

En la Tabla 3 se observan los resultados de los alfas de Cronbach para el constructo grupos de interés. La medición que se presenta incluye dos conjuntos. El primero de ellos muestra los resultados obtenidos al considerar dentro del constructo a los tres atributos (poder, legitimidad y urgencia) y la frecuencia de los intentos de influencia. El segundo muestra los resultados obtenidos considerando únicamente los tres atributos. Los valores hallados nos indican que en general el nivel de coherencia está entre aceptable y 
bueno, excepto para el grupo de interés formado por las Directivas de la Universidad y en menor medida el grupo Gobierno, que presentan valores por debajo de los recomendables. Conviene destacar que sólo se presentan los resultados para el grupo de interés de los Administrativos, dentro de los adicionales que propusieron los entrevistados, por ser el único entre nueve de ellos que alcanzó una relevancia ponderada significativa.

Los resultados de la Tabla 2 indican que el constructo atributos permite medir de forma aceptable los atributos de los grupos de interés (poder, legitimidad y urgencia, Mitchell et al., 1997). Se destacan en su orden, la urgencia y el poder. En la percepción de los líderes entrevistados, están más claros estos dos atributos. La urgencia como indicativo de la celeridad que imprime la rutina de los procesos a la labor de los decisores, cuando un grupo de interés así lo exige. De igual forma, también se manifiesta con claridad el atributo del poder de los grupos de interés en la percepción de los líderes, aunque en este estudio no se distingue la naturaleza de la fuente de ese poder (coercitiva, utilitaria o normativa, Mitchell et al., 1997).

Tabla 3: Alfas de Cronbach para el constructo grupos de interés

\begin{tabular}{|l|c|c|}
\hline Grupo de Interés & Alfa de Cronbach. Atributos+Frecuencia & Alfa de Cronbach . sólo Atributos \\
\hline Directivas de la Universidad & 0,443 & 0,277 \\
\hline Profesores & 0,689 & 0,550 \\
\hline Grupos de Investigación & 0,749 & 0,728 \\
\hline Empresas & 0,802 & 0,721 \\
\hline Estudiantes & 0,749 & 0,669 \\
\hline Sociedad en general & 0,736 & 0,652 \\
\hline Financiadores & 0,828 & 0,850 \\
\hline Gobierno & 0,479 & 0,512 \\
\hline Graduados & 0,819 & 0,803 \\
\hline Medio Ambiente & 0,783 & 0,791 \\
\hline Administrativos & 0,903 & 0,930 \\
\hline
\end{tabular}

El atributo de la legitimidad se encuentra un poco menos claro en la percepción de los líderes. La circunstancia puede obedecer a la coyuntura en la cual se llevó a cabo la medición, dada la existencia de una anormalidad académica en la cual varios grupos de interés promovieron el cese de actividades en dos de las tres universidades, mientras en una de ellas, adicionalmente presionaron a las instancias superiores del gobierno universitario para que modificara la terna de candidatos a Rector y convocara al proceso de consulta para su designación, por considerar que el proceso que se llevaba hasta entonces carecía de legitimidad plena y favorecía el continuismo. De todos modos, es importante destacar, que la legitimidad de algunos grupos de interés ganó fuerza al generar coaliciones con otros grupos interesados, y en este orden de ideas, se hicieron a los atributos que les hacían falta para ganar notoriedad y relevancia.

Por su parte, los resultados para el segundo constructo indican la existencia de once principales grupos de interés universitarios para las organizaciones estudiadas, los cuales se cuentan dentro de los sugeridos en la literatura particular sobre el tema (Duque Oliva, 2009; Vallaeys et al., 2009; Rodríguez Fernández, 2010). Los ocho grupos de interés que no alcanzaron una relevancia ponderada que permitiera su inclusión en el conjunto de grupos de interés para el cuestionario final, se puede entender como una respuesta a los factores contextuales (Osborn et al., 2002; Porter y McLaughlin, 2006) que indican cuáles grupos de interés hay que considerar y cuáles no. Incluso, tomando como referencia el trabajo de Vieira (2013), se presentó variación en la jerarquía en la cual se ubican los grupos de interés finales, siendo importante destacar que en el proceso de validación surgió de forma destacada el grupo de interés de los Administrativos, el cual no existía en los resultados de Vieira (2013) aunque si fueron incluidos por Caballero Fernández et al. (2007).

Tal como lo ilustra la Tabla 3, nueve de los once grupos de interés en consideración dentro del constructo que incluye a los atributos y la frecuencia de los intentos de influencia, obtuvieron alfas de Cronbach que permiten afirmar que son percibidos con claridad por parte de los líderes en las universidades. Para el caso de las Directivas de la Universidad y del Gobierno no puede hacerse esta afirmación. A fin de contrastar los resultados para este constructo, la segunda columna de la Tabla 3 ilustra los resultados obtenidos al excluir la variable frecuencia de los intentos de influencia. En este caso se aprecian resultados diferentes en comparación con los primeros, pero ratifican la claridad de la forma como son percibidos por los líderes directivos un amplio conjunto de grupos de interés, salvo que en esta ocasión aunque la claridad del Gobierno mejora, la de los profesores se deteriora. Ya para el caso de las Directivas de la Universidad, la claridad con la que este grupo es percibido desciende y se ratifica su condición de poca claridad. 
En el caso del alfa de Cronbach para los grupos de interés Directivas y Gobierno, cuyos valores están por debajo de lo aceptable, se considera este hecho como eminentemente contextual, además, es un indicativo de que no existe una noción clara y homogénea de la forma como se perciben estos grupos de interés en las universidades analizadas, como también lo indica un análisis factorial confirmatorio. Esta situación implica incertidumbre debido a la variabilidad que manifiestan los líderes directivos en la percepción de la naturaleza de cada uno de estos dos grupos, que para ellos lucen heterogéneos. Al ser las Directivas de las universidades los representantes del Gobierno, se encuentran en la frontera entre los grupos de interés internos y externos, situación que para este contexto particular, puede estar generando distorsión en la identificación de sus diferencias. Esto debido posiblemente a que primero, para las dos universidades más grandes, los entrevistados fungieron previamente como directivas de estas universidades; y segundo, a que para designar candidatos a ocupar cargos directivos, su elección pasa por los filtros de la cadena jerárquica encabezada por sus Consejos Superiores, en los cuales hay una significativa representación del Gobierno debido a su naturaleza pública.

De acuerdo con los resultado de los alfas de Cronbach se afirma que hay una fuerte coherencia interna y existe validez para el constructo grupos de interés. Aunque algunos alfas de Cronbach resulten menos fuertes que otros, al encontrarse en la frontera superior de la aceptabilidad, pueden considerarse incluidos dentro del conjunto general que manifiesta la coherencia interna del cuestionario, indicativo de que éste hace factible medir de manera eficaz la relevancia de los grupos de interés en las universidades estudiadas.

\section{CONCLUSIONES}

Con base en la discusión teórica presentada y teniendo en cuenta los resultados de la validación de los constructos presentados en el cuestionario, su análisis y discusión, se puede concluir lo siguiente:

Es el contexto particular de cada organización el que determina la composición de los grupos de interés que pueden resultar influyentes y por lo tanto relevantes, de acuerdo con la percepción de los actores que sean objeto de interés en cada investigación en particular. Desde el nivel de análisis individual, particularmente, desde la percepción de los líderes directivos entrevistados, el cuestionario desarrollado permite identificar los grupos de interés de las universidades públicas de la ciudad de Manizales (Colombia).

De acuerdo con los resultados obtenidos de los análisis estadísticos realizados, se puede afirmar que el cuestionario para valorar los constructos atributos y grupos de interés universitarios, cuenta con la consistencia interna y la fiabilidad de medida necesaria para llevar a cabo esta valoración en las organizaciones estudiadas.

En caso de que el nivel de análisis al cual se desee llevar a cabo el estudio cambie al de díadas, grupos o grupos de grupos, será necesario llevar a cabo el ajuste del cuestionario de modo que las preguntas se adapten al nivel de percepción que se desee indagar y que la relevancia sea un reflejo veraz de la percepción que se busca identificar.

\section{AGRADECIMIENTOS}

Los autores desean expresar su agradecimiento a la Universidad Nacional de Colombia, y en particular a la Facultad de Ingeniería y Arquitectura y a su Dirección de Investigación y Extensión, sin cuyo apoyo integral no hubiera sido posible adelantar la investigación de la cual surgió este artículo.

\section{REFERENCIAS}

Antonakis, J., y otros cinco autores, Methods for studying leadership, In The nature of leadership by Antonakis, J., Cianciolo, A.T. y Sternberg, R.J., pp 48-70 Sage Publications, Thousand Oaks (2004).

Ayuga-Téllez, E., González-García, C., Ortiz, M. A., \& Martínez-Falero, E. Diseño de un cuestionario para evaluar conocimientos básicos de estadística de estudiantes del último curso de ingeniería, doi: 10.4067/S0718-50062012000100004. Formación universitaria, (en línea), 5(1), 21-32 (2012).

Bass, B.M. The Bass handbook of leadership: theory, research, and managerial applications / Bernard M. Bass, with Ruth Bass. 4th edition, Free Press, New York (2008).

Blau, P.M., Exchange and power in social life, Wiley, New York (1964).

Caballero Fernández, G., J.M. García Vásquez, y M.A. Quintas Corredoira, La importancia de los stakeholders de la organización: un análisis empírico aplicado a la empleabilidad del alumnado de la universidad española, Investigaciones Europeas de Dirección y Economía de Empresas, ISSN: 1135-2523 
(en línea), 13(2), 13-32, 2007. http://www.redalyc.org/articulo.oa?id=274120280001. Acceso: 25 de enero (2015).

Delgado, M. L. Las comunidades de liderazgo de centros educativos. Educar, ISSN: 2014-8801, (en línea), (48), 9-21, 2012. http://www.raco.cat/index.php/Educar/article/viewFile/252990/339735. Acceso: 25 de enero (2015)

Delgado, M. L., Reche, M. P. C., Díaz, I. A., Lucena, F. J. H., \& Torres, J. M. T. Aportaciones actuales del liderazgo estudiantil en el contexto italiano: la Universidad de Bolonia. Educatio siglo XXI: Revista de la Facultad de Educación, ISSN: 1699-2105, (en línea), (29), 313-332, 2011. http://dialnet.unirioja.es/servlet/articulo?codigo=3719924. Acceso: 25 de enero (2015)

Dionne S.D., J.U. Chun, C.H. Andra Serban, F.J. Yammarino y W.D. Spangler, Article quality and publication impact via levels of analysis incorporation: An illustration with transformational/charismatic leadership, doi: doi:10.1016/j.leaqua.2012.10.003, The Leadership Quarterly, (en línea), 23, 1012-1042 (2012).

Duque Oliva, E. J., La gestión de la universidad como elemento básico del sistema universitario: una reflexión desde la perspectiva de los stakeholders, Innovar, ISSN: 2248-6968 (en línea), 19( ), 24-42, 2009. http://www.scielo.org.co/pdf/inno/v19s1/19s1a03.pdf. Acceso: 25 de enero (2015)

Freeman, R. E. A stakeholder theory of the modern corporation. Perspectives in Business Ethics Sie, 3, 144. 2001. http://academic.udayton.edu/LawrenceUlrich/Stakeholder\%20Theory.pdf. Acceso: 25 de enero (2015)

Gardner W.L., K.B. Lowe, T.W. Moss, K.T. Mahoney y C. Cogliser, Scholarly leadership of the study of leadership: A review of The Leadership Quarterly's second decade, 2000-2009, doi:10.1016/j.leaqua.2010.10.003, The Leadership Quarterly, (en línea), 21, 922-958 (2010).

Gastélum, G., Guedea, J. C., Viciana, J., \& Peinado, J. E. Composición Factorial de una Escala de Autoeficacia en el Ámbito del Trabajo en Equipo y Liderazgo en Universitarios de Ciencias de la Salud, doi: 10.4067/S0718-50062012000400006. Formación universitaria, (en línea), 5(4), 49-60 (2012).

Graen G.B. y M. Uhl-Bien, Relationship-based approach to leadership: Development of leader-member exchange (LMX) theory of leadership over 25 years: Applying a multi-level multi-domain perspective, doi:10.1016/1048-9843(95)90036-5, The Leadership Quarterly, (en línea), 6(2), 219-247 (1995).

Helmstadter G.C. Principles of psychological measurement. New York: Appleton-Century-Crofts, 1964.

Hernandez M., M.B. Eberly, B.J. Avolio y M.D. Johnson, The loci and mechanisms of leadership: Exploring a more comprehensive view of leadership theory, doi:10.1016/j.leaqua.2011.09.009, The Leadership Quarterly, (en línea), 22, 1165-1185 (2011).

Homans, G.C., Social behavior: Its elementary forms, Harcourt, Brace \& World, New York (1961).

Katz, D. y Kahn, R.L. Psicología social de las organizaciones, Trillas, México (1977).

Magness, V. Who are the stakeholders now? An empirical examination of the Mitchell, Agle and Wood Theory of Stakeholder Salience, doi: 10.1007/s10551-007-9610-2, Journal of Business Ethics, (en línea), 83 (2), 177-192 (2008).

May, T. Social Research. Issues, Methods and Process (4 ed.), Open University Press y McGraw Hill, Maidenhead (Inglaterra) (2011).

Mintzberg, H., Power in and around organizations, Prentice Hall, Englewood Cliffs (1983).

Mitchell, R.K., B.R. Agle, y D.J. Wood, Toward a theory of stakeholder identification and salience: defining the principle of who and what really counts, doi:10.5465/AMR.1997.9711022105, Academy of Management Review, (en línea), 22(4), 853-886 (1997).

Morales Rodríguez J.F. La teoría del intercambio social desde la perspectiva de Blau, Reis, ISSN 02105233 (en línea), 4,129-146, 1978. http://www.jstor.org/stable/40182727. Acceso: 25 de enero (2015)

Myllykangas, P., J. Kujala y H. Lehtima"ki, Analyzing the essence of stakeholder relationships: What do we need in addition to power, legitimacy, and urgency?, doi: 10.1007/s10551-011-0945-3, Journal of Business Ethics, (en línea), 96 (August), 65-72 (2010).

Nunnally, J., \& Bernstein, I. (1999). Teoría psicométrica. México: Trillas.

Osborn, R.N., J.B. Hunt y L.R. Jauch, Toward a contextual theory of Leadership, doi:10.1016/S10489843(02)00154-6, The Leadership Quarterly, (en línea), 13, 797-837 (2002). 
Pareja Fernández de la Reguera, J. A., López Núñez, J. A., El Homrani, M., \& Lorenzo Martín, R. El liderazgo en los estudiantes universitarios: una fructífera línea de investigación. Educar ISSN: 2014-8801 (en línea), (Vol. 48, pp. 0091-119), 2012. http://www.raco.cat/index.php/educar/article/viewArticle/252994/0. Acceso: 25 de enero (2015)

Phillips, E.M. y Pugh, D.S., La tesis doctoral. Un manual para doctorandos y sus directores, Bresca, Barcelona (2008).

Popper, K., En busca de un mundo mejor, Paidós, Barcelona (1994).

Porter, L.W. y G.B. McLaughlin, Leadership and the organizational context: Like the weather?, doi:10.1016/j.leaqua.2006.10.002, The Leadership Quarterly: 17 (6), 559-576 (2006).

Reche, M.P.C., Delgado, M. L., \& Martínez, T. S. El liderazgo estudiantil en la Universidad de Granada desde una dimensión introspectiva. Bordón. Revista de pedagogía, ISSN: 0210-5934, (en línea), 61(1), 109130, 2009. http://dialnet.unirioja.es/descarga/articulo/2968992.pdf. Acceso: 25 de enero (2015)

Reche, M.P.C., Delgado, M. L., \& Martínez, T. S. Evaluación de la representación estudiantil en la Universidad desde un enfoque de género: diseño de un cuestionario. Enseñanza \& Teaching: Revista interuniversitaria de didáctica, ISSN: 2386-3927, (en línea), (26), 137-164, 2008. http://rca.usal.es/index.php/0212-5374/article/view/691. Acceso: 25 de enero (2015)

Rodríguez Fernández, J. M., Responsabilidad Social Universitaria: Del discurso simbólico a los desafíos reales. In Responsabilidad Social Universitaria by Cuesta González, M., Cruz Ayuso, C. y Rodríguez Fernández, J.M (Ed.), pp 3-24 Netbiblo. (2010).

Schneider, M., A Stakeholder Model of Organizational Leadership, doi:10.1287/orsc.13.2.209.531, Organization Science, (en línea), 13(2), 209-220 (2002).

Schriesheim C.A., S.L. Castro , X. Zhou y F.J. Yammarino, The folly of theorizing " $A$ " but testing "B" $A$ selective level-of-analysis review of the field and a detailed Leader-Member Exchange illustration, doi:10.1016/S1048-9843(01)00095-9, The Leadership Quarterly, (en línea), 12, 515-551 (2001).

Searle, J.R., La construcción de la realidad social, Paidós, Barcelona (1997).

Searle, J.R., Libertad y neurobiología. Reflexiones sobre el libre albedrío y el poder político, Paidos, Barcelona (2005).

Stentz J.E., V.L. Plano Clark y G.S. Matkin, Applying mixed methods to leadership research: A review of current practices, doi:10.1016/j.leaqua.2012.10.001, The Leadership Quarterly, (en línea), 23, 1173-1183 (2012).

Torres, J. M. T., Núñez, J. A. L., \& Martín, M. E. L. Análisis y descripción de las percepciones del liderazgo resiliente y liderazgo distribuido en torno al ejercicio directivo (2.0) como posibilidad para aprehender y transformar las instituciones educativas. REICE: Revista Electrónica Iberoamericana sobre Calidad, Eficacia y Cambio en Educación, ISSN: 1696-4713, (en línea), 9(3), 13-29, 2011. http://dialnet.unirioja.es/descarga/articulo/3932577.pdf. Acceso: 25 de enero (2015)

Toro, I.D. y Parra, R.D., Fundamentos epistemológicos de la investigación y la metodología de investigación - cualitativa/cuantitativa, Fondo Editorial Universidad Eafit, Medellín (2010).

Vallaeys, F., de la Cruz, C., y Sasia, P. (2009), Responsabilidad Social Universitaria. Manual de Primeros Pasos, $1^{\underline{a}}$ edición, Mc Graw Hill Interamericana Editores S.A. de C.V., México (2009).

Vieira, J.A., The socially responsible management in Colombian public universities. Case study: the research function in public universities at Manizales (Colombia), Tesis de Doctorado, Université de Rouen Laboratoire du NIMEC. Rouen-France (2013).

Vroom, V.H. y A.G. Jago, Situation effects and levels of analysis in the study of leader participation, doi:10.1016/1048-9843(95)90033-0, The Leadership Quarterly, (en línea), 6 (2), 169-181 (1995).

Yammarino F.J., S.D. Dionne, J.U. Chun y F. Dansereau, Leadership and levels of analysis: $A$ state-of-thescience review, doi:10.1016/j.leaqua.2005.09.002, The Leadership Quarterly, (en línea), 16, 879-919 (2005).

Yukl, G., Leadership in organizations, seventh edition. Prentice Hall, New Jersey (2010). 